\title{
HATS-59b,c: A Transiting Hot Jupiter and a Cold Massive Giant Planet around a Sunlike Star
}

Sarkis, P.; Henning, Th.; Hartman, J. D.; Bakos, G. A.; Brahm, R.; Jordan, A.; Bayliss, D.; Mancini, L.; Espinoza, N.; Rabus, M.

Total number of authors:

30

Published in:

Astronomical Journal

Link to article, DOI:

10.3847/1538-3881/aade54

Publication date:

2018

Document Version

Publisher's PDF, also known as Version of record

Link back to DTU Orbit

Citation $(A P A)$ :

Sarkis, P., Henning, T., Hartman, J. D., Bakos, G. A., Brahm, R., Jordan, A., Bayliss, D., Mancini, L., Espinoza, N., Rabus, M., Csubry, Z., Bhatti, W., Penev, K., Zhou, G., Bento, J., Tan, T. G., Arriagada, P., Butler, R. P., Crane, J. D., ... Sari, P. (2018). HATS-59b,c: A Transiting Hot Jupiter and a Cold Massive Giant Planet around a Sunlike Star. Astronomical Journal, 156(5), [216]. https://doi.org/10.3847/1538-3881/aade54

\section{General rights}

Copyright and moral rights for the publications made accessible in the public portal are retained by the authors and/or other copyright owners and it is a condition of accessing publications that users recognise and abide by the legal requirements associated with these rights.

- Users may download and print one copy of any publication from the public portal for the purpose of private study or research.

- You may not further distribute the material or use it for any profit-making activity or commercial gain

- You may freely distribute the URL identifying the publication in the public portal 


\title{
HATS-59b,c: A Transiting Hot Jupiter and a Cold Massive Giant Planet around a Sun- like Star*
}

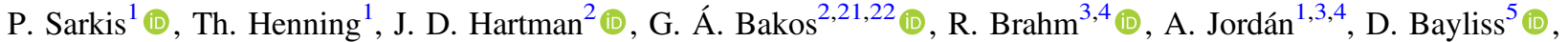 \\ L. Mancini ${ }^{1,6,7}$ (D), N. Espinoza ${ }^{1,3,4}$, M. Rabus ${ }^{1,3}$, Z. Csubry ${ }^{2}$, W. Bhatti ${ }^{2}$ (iD, K. Penev ${ }^{8}$ (D), G. Zhou ${ }^{9}$, J. Bento ${ }^{10}$, T. G. Tan ${ }^{11}$, \\ P. Arriagada ${ }^{12}$ (D), R. P. Butler ${ }^{13}$ (D), J. D. Crane ${ }^{13}$ (D), S. Shectman ${ }^{13}$, C. G. Tinney ${ }^{14,15}$ (D), D. J. Wright ${ }^{14,15}$, B. Addison ${ }^{16}$, \\ S. Durkan ${ }^{17}$ (D), V. Suc ${ }^{3}$ (D), L. A. Buchhave ${ }^{18}$ (D), M. de Val-Borro ${ }^{19}$, J. Lázár ${ }^{20}$, I. Papp ${ }^{20}$, and P. Sári ${ }^{20}$ \\ ${ }_{1}^{1}$ Max Planck Institute for Astronomy, Heidelberg, Germany; sarkis@mpia.de \\ ${ }^{2}$ Department of Astrophysical Sciences, Princeton University, NJ 08544, USA \\ ${ }^{3}$ Instituto de Astrofísica, Facultad de Física, Pontificia Universidad Católica de Chile, Av. Vicuña Mackenna 4860, 7820436 Macul, Santiago, Chile \\ ${ }^{4}$ Millennium Institute of Astrophysics, Av. Vicuña Mackenna 4860, 7820436 Macul, Santiago, Chile \\ ${ }^{5}$ Department of Physics, University of Warwick, Coventry CV4 7AL, UK \\ ${ }^{6}$ Department of Physics, University of Rome Tor Vergata, Via della Ricerca Scientifica 1, I-00133 Rome, Italy \\ ${ }^{7}$ INAF-Astrophysical Observatory of Turin, via Osservatorio 20, I-10025 Pino Torinese, Italy \\ ${ }^{8}$ Physics Department, University of Texas at Dallas, 800 W Campbell Rd. MS WT15, Richardson, TX 75080, USA \\ ${ }^{9}$ Harvard-Smithsonian Center for Astrophysics, Cambridge, MA 02138, USA \\ ${ }^{10}$ Research School of Astronomy and Astrophysics, Australian National University, Canberra, ACT 2611, Australia \\ ${ }^{11}$ Perth Exoplanet Survey Telescope, Perth, Australia \\ 12 Department of Terrestrial Magnetism, Carnegie Institution for Science, Washington, DC 20015, USA \\ 13 The Observatories of the Carnegie Institution for Science, 813 Santa Barbara St., Pasadena, CA 91101, USA \\ ${ }^{14}$ Australian Centre for Astrobiology, School of Physics, University of New South Wales, NSW 2052, Australia \\ ${ }_{15}$ Exoplanetary Science at UNSW, School of Physics, University of New South Wales, NSW 2052, Australia \\ ${ }^{16}$ Mississippi State University, Department of Physics \& Astronomy, Hilbun Hall, Starkville, MS 39762, USA \\ ${ }_{17}$ Astrophysics Research Centre, Queens University, Belfast, Belfast, Northern Ireland, UK \\ ${ }^{18}$ DTU Space, National Space Institute, Technical University of Denmark, Elektrovej 328, DK-2800 Kgs. Lyngby, Denmark \\ ${ }^{19}$ Astrochemistry Laboratory, Goddard Space Flight Center, NASA, 8800 Greenbelt Rd., Greenbelt, MD 20771, USA \\ ${ }^{20}$ Hungarian Astronomical Association, 1451 Budapest, Hungary \\ Received 2018 May 13; revised 2018 August 24; accepted 2018 August 29; published 2018 October 18
}

\begin{abstract}
We report the first discovery of a multi-planetary system by the HATSouth network, HATS-59b,c, a planetary system with an inner transiting hot Jupiter and an outer cold massive giant planet, which was detected via radial velocity. The inner transiting planet, HATS-59b, is on an eccentric orbit with $e=0.129 \pm 0.049$, orbiting a $V=13.951 \pm 0.030$ mag solar-like star $\left(M_{\star}=1.038 \pm 0.039 M_{\odot}\right.$ and $\left.R_{\star}=1.036 \pm 0.067 R_{\odot}\right)$ with a period of $5.416081 \pm 0.000016$ days. The outer companion, HATS-59c is on a circular orbit with $m \sin i=12.70 \pm$ $0.87 M_{\mathrm{J}}$ and a period of $1422 \pm 14$ days. The inner planet has a mass of $0.806 \pm 0.069 M_{\mathrm{J}}$ and a radius of $1.126 \pm 0.077 R_{\mathrm{J}}$, yielding a density of $0.70 \pm 0.16 \mathrm{~g} \mathrm{~cm}^{-3}$. Unlike most planetary systems that include only a single hot Jupiter, HATS-59b,c includes, in addition to the transiting hot Jupiter, a massive outer companion. The architecture of this system is valuable for understanding planet migration.
\end{abstract}

Key words: photometric - planetary systems - stars: individual (HATS-59) - techniques: spectroscopic

Supporting material: machine-readable table

\section{Introduction}

During the past decade, the number of exoplanets has increased steadily and by now more than 3500 exoplanets have been

\footnotetext{
* The HATSouth network is operated by a collaboration consisting of Princeton University (PU), the Max Planck Institute für Astronomie (MPIA), the Australian National University (ANU), and the Pontificia Universidad Católica de Chile (PUC). The station at Las Campanas Observatory (LCO) of the Carnegie Institute is operated by PU in conjunction with PUC, the station at the High Energy Spectroscopic Survey (H.E.S.S.) site is operated in conjunction with MPIA, and the station at Siding Spring Observatory (SSO) is operated jointly with ANU. This paper includes data gathered with $6.5 \mathrm{~m}$ Magellan Telescopes located as Las Campanas Observatory, Chile, and the MPG $2.2 \mathrm{~m}$, the NTT, and the Euler $1.2 \mathrm{~m}$ telescopes at the ESO Observatory in La Silla. This paper uses observations obtained with facilities of the Las Cumbres Observatory Global Telescope. Based in part on observations made with the $3.9 \mathrm{~m}$ Anglo-Australian Telescope and the ANU $2.3 \mathrm{~m}$ Telescope both at SSO. Based in part on observations made with the facilities of the Las Cumbres Observatory Global Telescope, the Perth Exoplanet Survey Telescope, and the Nordic Optical Telescope.

${ }^{21}$ Packard Fellow.

22 MTA Distinguished Guest Fellow, Konkoly Observatory.
}

statistically validated. Exoplanets are very common and have a wide variety of properties (for a review, see Winn \& Fabrycky 2015), which offer a unique opportunity to constrain their formation and evolution (Mordasini et al. 2016; Jin \& Mordasini 2018). Hot Jupiters, i.e., gas giant planets on short orbital periods, still pose many challenges for planet formation models. It is believed that such planets formed beyond the iceline, several au from the central star, and migrated inwards through interactions with the disk (e.g., Lin et al. 1996). However, disk migration predicts circular and aligned orbits (e.g., Goldreich \& Tremaine 1980; Artymowicz 1993) and cannot explain the existence of several hot Jupiters that have been found on retrogade or misaligned orbits (for a review see Winn \& Fabrycky 2015). Alternative scenarios have been thus proposed, which involve interactions with a third distant body or planet-planet scattering that can result in eccentric and misaligned orbits (Kozai 1962; Lidov 1962; Nagasawa et al. 2008; Li et al. 2014; Petrovich 2015).

One approach to put constraints on the different migration mechanisms is to measure the spin-orbit alignment via the 
Rossiter-McLaughlin effect (e.g., Queloz et al. 2000; Zhou et al. 2015). Another approach is to search for planetary or stellar companions at large separations, which could have influenced the dynamical evolution of the inner planet. Knutson et al. (2014) performed a long-term radial velocity monitoring of 51 systems known to host a hot Jupiter, with the goal to detect further planetary companions. They estimated an occurrence rate of $51 \% \pm 10 \%$ for companions with masses between 1 and $13 M_{\mathrm{J}}$ and orbital semimajor axes between 1 and $20 \mathrm{au}$. Ngo et al. (2015) presented the results on searching for stellar companions around 50 out of the 51 selected systems from Knutson et al. (2014) study. They corrected for survey incompleteness and reported a stellar companion fraction of $48 \% \pm 9 \%$. Combining the results of both studies, $\mathrm{Ngo}$ et al. (2015) estimated that $72 \% \pm 16 \%$ of hot Jupiters are part of multi-planet and/or multi-star systems.

In this work, we report the discovery of HATS-59b,c, the first multi-planet system detected by the HATSouth survey (Bakos et al. 2013). The star hosts an inner hot Jupiter detected via its transits and an outer cold massive giant planet detected via the radial velocity variations of the host star. The possibility of additional outer planetary companions to transiting hot Jupiter has been proposed by, e.g., Rabus et al. (2009) and in fact, there have been only a few transiting planets with an outer planetary companion for which a full orbit was detected via radial velocity, such as HAT-P-13b,c (Bakos et al. 2009), HAT-P-17b,c (Howard et al. 2012), Kepler-424b,c (Endl et al. 2014), WASP-41b,c (Neveu-VanMalle et al. 2016), WASP-47b,c (Hellier et al. 2012; Becker et al. 2015; NeveuVanMalle et al. 2016), and WASP-53b,c (Triaud et al. 2017). Among all the systems with a transiting hot Jupiter known to have outer companions, HAT-P-13 c and WASP-53b,c are the only massive planetary companions with a minimum mass greater than HATS-59 c. The few detections of companions around transiting planets is due, to some extent, by the lack of radial velocity follow-up observations. Hot Jupiters in multiplanet systems provide a unique opportunity to place observational constraints on migration models and also could be used to probe the tidal love number of the hot Jupiter (Buhler et al. 2016; Hardy et al. 2017), which in turn constrains the planetary interior structure (Batygin et al. 2009). Therefore, monitoring these systems is very interesting for planet formation and interior structure models.

The paper is structured as follows: In Section 2, we show the planetary signal detected by the HATSouth network and present the photometric and spectroscopic follow-up observations that allowed us to characterize the system. In Section 3, we derive the stellar parameters and jointly model the data to derive the planetary parameters. Our results are finally summarized in Section 4.

\section{Observations}

\subsection{Photometry}

\subsubsection{Photometric Detection}

The HATS-59 system was identified by the HATSouth instruments as potentially hosting a transiting planet. The star (Table 3) was observed between UT 2010 January 19 and UT 2010 August 10 using the HS-1, HS-3, and HS-5 units at the Las Campanas Observatory (LCO) in Chile, the H.E.S.S. site in Namibia, and the Siding Springs Observatory (SSO) in Australia, respectively. A total of 3113, 4690 and 658 of useful
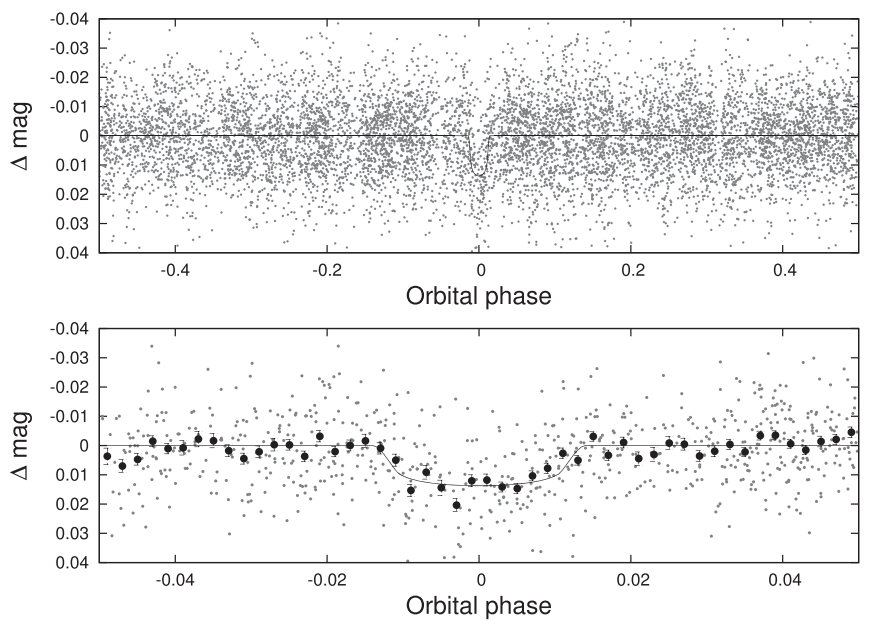

Figure 1. The discovery light curve of HATS-59 phase-folded with a period of $P=5.4160810$ days (see Section 3). The lower panel shows the transit where the filled black points show the light curve binned in phase with a bin size of 0.002 . The solid lines in both panels show the best-fit transit model.

Table 1

Differential Photometry of HATS-59

\begin{tabular}{lrcccc}
\hline \hline $\begin{array}{l}\text { BJD } \\
(2400000+)\end{array}$ & \multicolumn{1}{c}{ Mag $^{\mathrm{a}}$} & $\sigma_{\text {Mag }}$ & $\begin{array}{c}\text { Mag } \\
(\text { orig })^{\mathrm{b}}\end{array}$ & Filter & Instrument \\
\hline 55372.26299 & -0.01448 & 0.00725 & $\cdots$ & $r$ & HS/G563.1 \\
55274.77568 & 0.01224 & 0.00650 & $\cdots$ & $r$ & HS/G563.1 \\
55296.44071 & 0.01384 & 0.00668 & $\cdots$ & $r$ & HS/G563.1 \\
55274.77891 & -0.01225 & 0.00628 & $\cdots$ & $r$ & HS/G563.1 \\
55296.44428 & -0.00169 & 0.00659 & $\cdots$ & $r$ & HS/G563.1 \\
55274.78240 & -0.01307 & 0.00627 & $\cdots$ & $r$ & HS/G563.1 \\
55296.44754 & -0.00042 & 0.00652 & $\cdots$ & $r$ & HS/G563.1 \\
55274.78561 & 0.00435 & 0.00643 & $\cdots$ & $r$ & HS/G563.1 \\
55296.45080 & -0.00521 & 0.00660 & $\cdots$ & $r$ & HS/G563.1 \\
55372.27744 & 0.00356 & 0.00771 & $\cdots$ & $r$ & HS/G563.1
\end{tabular}

Notes. The data are also available on the HATSouth website at http://www. hatsouth.org.

${ }^{\text {a }}$ The out-of-transit level has been subtracted. For the HATSouth light curve (rows with "HS" in the Instrument column), these magnitudes have been detrended using the EPD and TFA procedures prior to fitting a transit model to the light curve. The magnitudes of the follow-up light curves (rows with an Instrument other than "HS") have been detrended with the EPD procedure, which was carried out simultaneously with the transit fit.

${ }^{b}$ Raw magnitude values for the follow-up light curve without applying the EPD procedure.

(This table is available in its entirety in machine-readable form.)

images were obtained with the HS-1, HS-3, and HS-5 telescopes, respectively, using the Sloan $r$ filter with an exposure time of $240 \mathrm{~s}$.

Similar to previous HATSouth discoveries, all the photometry data were reduced to trend-filtered light curves using the aperture photometry pipeline described by Penev et al. (2013). Systematic variations were removed using the External Parameter Decorrelation (EPD; Bakos et al. 2010) and the Trend Filtering Algorithm (TFA; Kovács et al. 2005). Then a transit search was performed using the Box Least Squares (BLS; Kovács et al. 2002) fitting algorithm and a period of 5.4161 was detected (Figure 1; the data is provided in Table 1). The rms scatter after subtracting the best-fit model transit is $0.012 \mathrm{mag}$. The star was then flagged as a planet-host candidate 


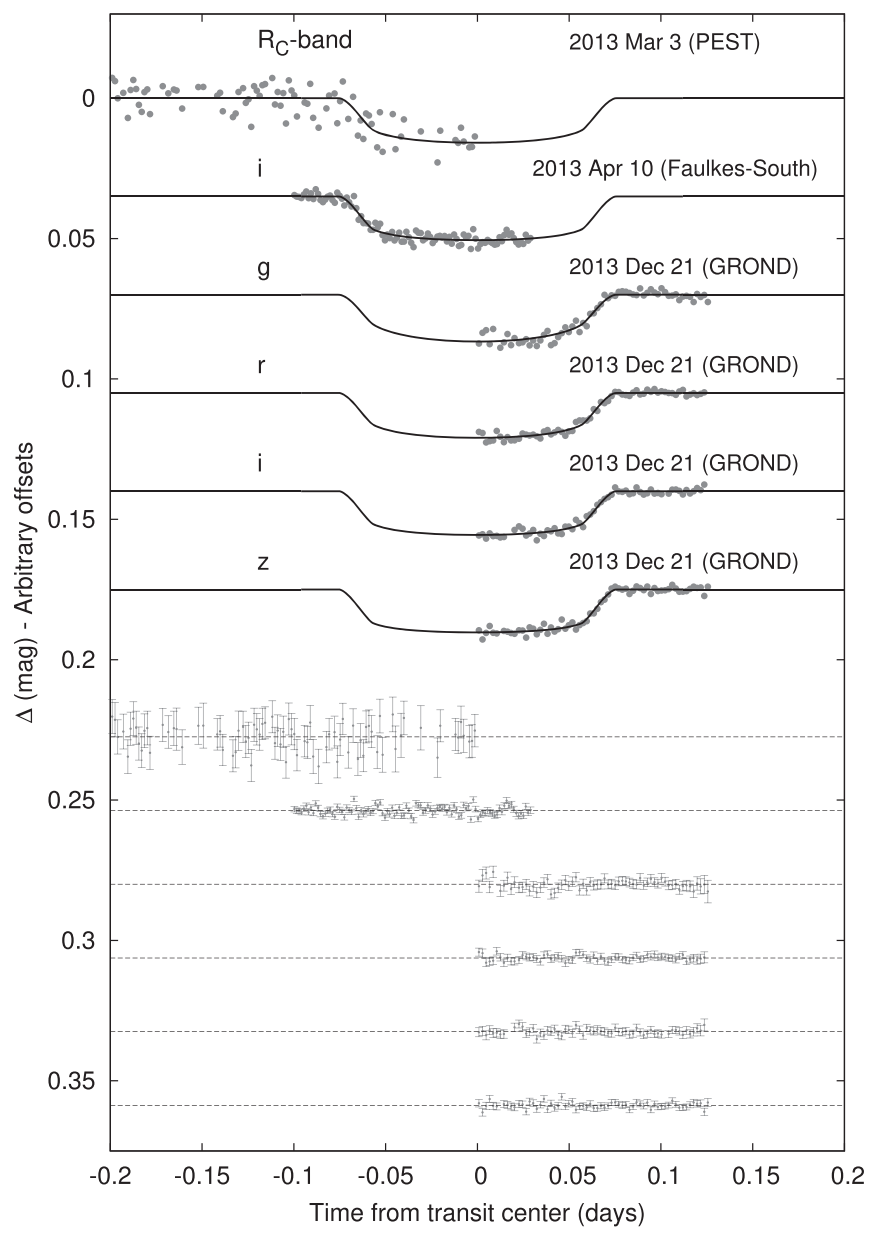

Figure 2. Unbinned follow-up transit light curve of HATS-59 compared to the best-fit transit model along with the residuals. The facilities and filters used, and the dates of each event are listed.

and approved for further follow-up photometric and spectroscopic observations.

\subsubsection{Photometric Follow Up}

In order to confirm that the transit signals detected in the discovery light curve are due to a transiting planet, we obtained photometric follow-up observations of three transit events. These light curves allow us to refine the ephemeris of the system and to determine precise parameters of the system. All the photometric data are provided in Table 1 and the follow-up light curves are shown in Figure 2 along with the best-fit model and residuals.

An ingress was observed with the $0.3 \mathrm{~m}$ Perth Exoplanet Telescope (PEST) on 2013 March 3, using the $R_{C}$ filter. The photometric precision of the light curve was $5.0 \mathrm{mmag}$ with a cadence of $130 \mathrm{~s}$. Another ingress was observed on 2013 April 10 using the Faulkes Telescope South (FTS), which is a fully automated telescope operated as part of the Las Cumbres Observatory Global Telescope (LCOGT; Brown et al. 2013). The transit was observed in the $i$-band filter achieving a photometric precision of $1.6 \mathrm{mmag}$ with a cadence of $113 \mathrm{~s}$. An egress was obtained on 2013 December 21 with the multiband imager GROND (Greiner et al. 2008), mounted on the $2.2 \mathrm{~m}$ telescope in La Silla Observatory, using four different filters $(g, r, i, z)$. The light curve had a precision of $1.7 \mathrm{mmag}$ in the $g$ band, 1.0 mmag in $r, 1.1 \mathrm{mmag}$ in $i$, and $1.1 \mathrm{mmag}$ in $z$, with a cadence of $168 \mathrm{~s}$. The details of the data reduction for these facilities are described in Penev et al. (2013), Mohler-Fischer et al. (2013), and Zhou et al. (2014a).

\subsection{Spectroscopic Observations}

HATS-59 was spectroscopically observed between 2011 April and 2016 March to confirm the planetary nature of the transit signals and to estimate the mass and therefore the density of the planet. Furthermore, the long radial velocity (RV) monitoring of the star allowed us to detect an outer companion with a longer orbital period than the transiting planet. We present the RV used to characterize the system in Figure 5 and provide the data in Table 2.

\subsubsection{Reconnaissance Spectroscopy}

Reconnaissance low-resolution spectroscopic follow-up observations are important to rule out various false positive scenarios, such as a primary giant star, or large RV variations indicating that the transiting object is itself a star. Reconnaissance spectroscopic observations were carried out with WiFeS (Dopita et al. 2007), a spectrograph mounted on the ANU $2.3 \mathrm{~m}$ telescope. We obtained a single $R=3000$ spectrum to estimate the stellar atmospheric parameters $T_{\text {eff } \star}$, $[\mathrm{Fe} / \mathrm{H}]$, and $v \sin i$ and were used to confirm that the star is a dwarf. In order to rule out large RV variations (at the level of $\sim 2 \mathrm{~km} \mathrm{~s}^{-1}$ ), we obtained 7 spectra with a resolution of $R=$ 7000. The spectra were extracted and reduced following Bayliss et al. (2013). Another reconnaissance spectrum was observed with the FIES spectrograph at the Nordic Optical Telescope (Telting et al. 2014), where it was reduced following Buchhave et al. (2010). We did not find large RV variations and thus ruled out the possibility that this system might be an eclipsing binary displaying a large radial velocity amplitude. We therefore proceeded with acquiring high-precision RV observations to characterize the system.

\subsubsection{High-precision Radial Velocities}

We carried out an intensive RV follow-up campaign to measure, with high precision, the semi-amplitude of the RV variations due to the transiting planet. The RV observations showed variations in phase with the transit ephemeris of the interior planet. They, additionally, showed evidence for a large amplitude sinusoidal variation with a period of $\sim 1400$ days. We next describe the observations and the data reduction of all the spectrographs used in this analysis.

We obtained nine spectra with the CORALIE spectrograph (Queloz et al. 2001) at the Euler $1.2 \mathrm{~m}$ telescope at La Silla. We also obtained five spectra with the Planet Finder Spectrograph (PFS; Crane et al. 2010) on the Magellan Clay $6.5 \mathrm{~m}$ telescope and seven spectra with CYCLOPS on the $3.9 \mathrm{~m}$ Anglo-Australian Telescope. Most of the spectra used in this analysis, most importantly for the discovery of the second outer companion, were obtained with FEROS on the MPG $2.2 \mathrm{~m}$ (Kaufer \& Pasquini 1998) in La Silla Observatory. Twenty-four spectra were acquired with FEROS, which is a high-resolution echelle spectrograph (Kaufer \& Pasquini 1998). All the spectra acquired with FEROS and CORALIE were reduced, extracted, and analyzed using the CERES pipeline (Brahm et al. 2017a). The radial velocities of the PFS spectra calibrated with an I2-cell, were computed by matching a template spectrum. For more information, we refer the reader to Butler et al. (1996). Details on 
Table 2

Relative Radial Velocities and Bisector Span Measurements of HATS-59

\begin{tabular}{|c|c|c|c|c|c|c|}
\hline $\begin{array}{l}\text { BJD } \\
(2450000+)\end{array}$ & $\begin{array}{c}\mathrm{RV}^{\mathrm{a}} \\
\left(\mathrm{m} \mathrm{s}^{-1}\right)\end{array}$ & $\begin{array}{c}\sigma_{\mathrm{RV}}^{\mathrm{b}} \\
\left(\mathrm{m} \mathrm{s}^{-1}\right)\end{array}$ & $\begin{array}{c}\mathrm{BS} \\
\left(\mathrm{m} \mathrm{s}^{-1}\right)\end{array}$ & $\sigma_{\mathrm{BS}}$ & Phase & Instrument \\
\hline 5722.48192 & -156.41 & 34.00 & -25.0 & 68.0 & 0.163 & FEROS \\
\hline 5725.50346 & 72.59 & 41.00 & -64.0 & 82.0 & 0.721 & FEROS \\
\hline 5737.51083 & -49.41 & 31.00 & 7.0 & 62.0 & 0.938 & FEROS \\
\hline 5738.54029 & -166.41 & 33.00 & -52.0 & 66.0 & 0.128 & FEROS \\
\hline 5754.47565 & -35.41 & 48.00 & -29.0 & 96.0 & 0.070 & FEROS \\
\hline 5934.15946 & 104.14 & 109.00 & $\ldots$ & $\ldots$ & 0.246 & CYCLOPS \\
\hline 5934.86914 & 72.59 & 33.00 & 8.0 & 66.0 & 0.377 & FEROS \\
\hline 5936.80355 & 219.59 & 27.00 & 4.0 & 54.0 & 0.735 & FEROS \\
\hline 5938.23445 & 22.14 & 177.00 & $\ldots$ & $\ldots$ & 0.999 & CYCLOPS \\
\hline 5938.87128 & 69.59 & 30.00 & -11.0 & 60.0 & 0.116 & FEROS \\
\hline 5939.81042 & 39.59 & 31.00 & -6.0 & 62.0 & 0.290 & FEROS \\
\hline 6057.03928 & 318.14 & 27.00 & $\ldots$ & $\ldots$ & 0.934 & CYCLOPS \\
\hline 6059.00663 & 65.14 & 73.00 & $\ldots$ & $\ldots$ & 0.298 & CYCLOPS \\
\hline 6059.08191 & 46.14 & 30.00 & $\ldots$ & $\ldots$ & 0.312 & CYCLOPS \\
\hline 6375.71072 & 224.59 & 24.00 & 87.0 & 48.0 & 0.773 & FEROS \\
\hline 6376.71477 & 101.59 & 26.00 & 237.0 & 52.0 & 0.958 & FEROS \\
\hline 6424.70951 & 229.59 & 40.00 & -90.0 & 80.0 & 0.820 & FEROS \\
\hline 6427.67642 & 54.59 & 69.00 & -123.0 & 138.0 & 0.367 & FEROS \\
\hline 6464.53773 & -119.61 & 50.00 & 43.0 & 100.0 & 0.173 & Coralie \\
\hline 6465.53888 & 85.39 & 43.00 & 65.0 & 86.0 & 0.358 & Coralie \\
\hline 6694.77700 & -71.73 & 6.28 & $\ldots$ & $\ldots$ & 0.684 & PFS \\
\hline 6696.82969 & -214.95 & 4.90 & $\ldots$ & $\ldots$ & 0.063 & PFS \\
\hline 6697.75879 & -248.73 & 4.86 & $\cdots$ & $\ldots$ & 0.234 & PFS \\
\hline 6700.82214 & -101.76 & 5.72 & $\ldots$ & $\ldots$ & 0.800 & PFS \\
\hline 7185.59484 & -117.41 & 19.00 & -27.0 & 38.0 & 0.306 & FEROS \\
\hline 7462.66518 & 204.59 & 11.00 & -28.0 & 22.0 & 0.464 & FEROS \\
\hline 7463.86306 & 302.59 & 16.00 & -37.0 & 32.0 & 0.685 & FEROS \\
\hline 7464.73538 & 254.59 & 14.00 & -29.0 & 28.0 & 0.846 & FEROS \\
\hline
\end{tabular}

Notes.

${ }^{\mathrm{a}}$ Relative RVs, with $\gamma_{R V}$ subtracted.

${ }^{\mathrm{b}}$ Internal errors not accounting for astrophysical/instrumental jitter.

the data reduction and analysis are described in previous HATSouth discovery papers, e.g., Jordán et al. (2014), Zhou et al. (2014b), Hartman et al. (2015). For details of the data reduction of CYCLOPS spectra, see Penev et al. (2013).

\subsection{Lucky Imaging}

High spatial resolution imaging were obtained as part of the follow-up campaign using the Astralux Sur camera (Hippler et al. 2009) on the New Technology Telescope (NTT), at La Silla Observatory in Chile. The lucky imaging observations are useful to identify close stellar companions that could affect the transit depth. The observations were carried out with the SDSS $z^{\prime}$ filter on 2015 December 23 and reduced following Espinoza et al. (2016) but we used instead the plate scale derived in Janson et al. (2017) of 15.2 mas pixel $^{-1}$, which is a better estimate than the one estimated in our previous work. Figure 3 shows the final reduced image and Figure 4 shows the contrast curve, where no resolved companion is detected within $2^{\prime \prime}$.

\section{Analysis}

\subsection{Properties of the Parent Star}

It is important to characterize the host star in order to measure precise planetary parameters. We used ZASPE (Brahm et al. 2017b) to get an initial estimate of the atmospheric parameters $\left(T_{\text {eff } \star}[\mathrm{Fe} / \mathrm{H}] v \sin i\right.$, and $\left.\log g_{\star}\right)$. The parameters were determined using the FEROS spectra, which 


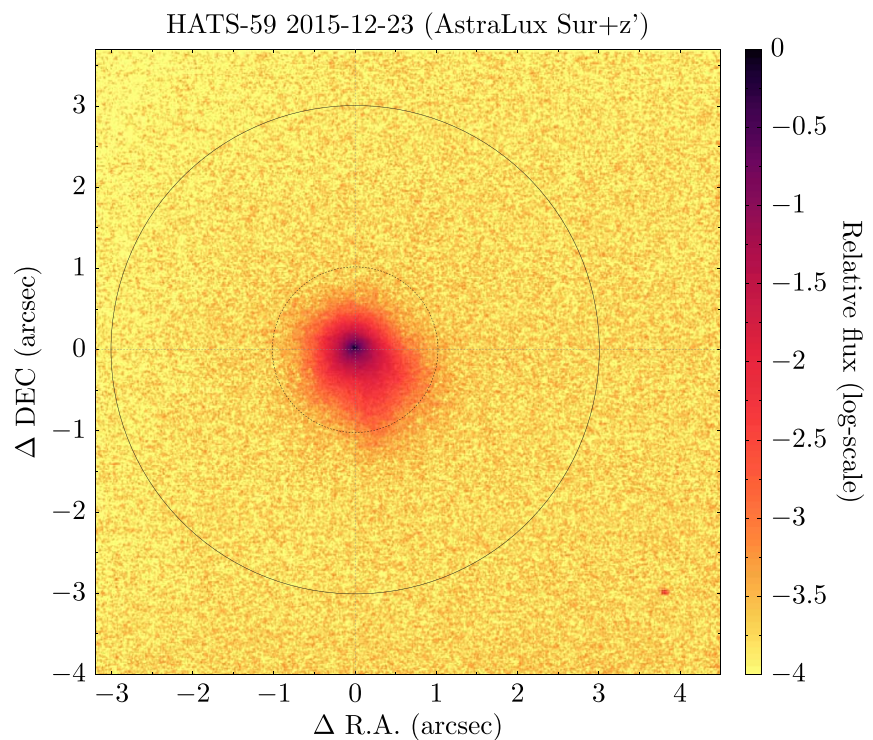

Figure 3. Astralux Sur lucky image of HATS-59 using $z^{\prime}$. Circles of $1^{\prime \prime}$ and $3^{\prime \prime}$ radii are shown. No neighboring companion is detected within $2^{\prime \prime}$.

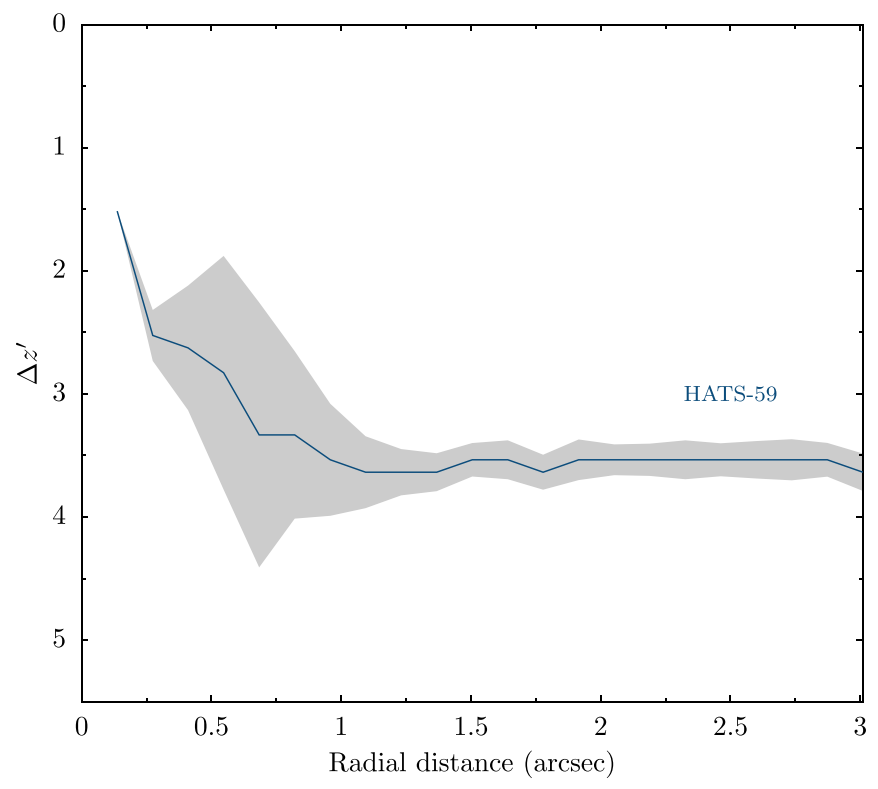

Figure 4. Contrast curve for of HATS-59 using the Astralux Sur $z^{\prime}$ observations. Gray bands show the uncertainty given by the scatter in the contrast in the azimuthal direction at a given radius.

were co-added to obtain a high signal-to-noise ratio spectrum. ZASPE determines the stellar parameters via least-squares minimization against a grid of synthetic spectra in the spectral regions most sensitive to changes in the parameters $(5000 \AA$ and $6000 \AA$ ).

We then followed Sozzetti et al. (2007) to determine the fundamental stellar parameters $\left(M_{\star}, R_{\star}, L_{\star}\right.$, age, etc.). In particular, we used the stellar density $\rho_{\star}$ determined from the photometric light curve, combined with the $T_{\text {eff } \star}$ and $[\mathrm{Fe} / \mathrm{H}]$ measurements, to characterize the host star. The parameters were obtained by combining the spectroscopic and photometric parameters with the Yonsei-Yale stellar evolution models $\left(\mathrm{Y}^{2}\right.$; Yi et al. 2001). This provided a revised estimate of $\log g_{\star}$, which was fixed in a second iteration of ZASPE that returned the final values of the stellar parameters.
We estimate a mass of $1.038 \pm 0.039 M_{\odot}$ and a radius of $1.036 \pm 0.067 R_{\odot}$. HATS-59 is at a reddening-corrected distance of $630 \pm 43 \mathrm{pc}$. The distance estimated using isochrone fitting is in agreement with the distance estimated using Gaia data. Figure 6 shows the location of the star on the $T_{\text {eff } \star}-\rho_{\star}$ diagram and the stellar parameters are provided in Table 3 .

\subsection{Excluding Blend Scenarios}

It is important to perform a blend analysis to confirm the planetary nature of the transiting signal and to rule out a stellar eclipsing binary system as a cause of the signal. Using the photometric data, the blend analysis was carried out following Hartman et al. (2012). We find that although blended stellar eclipsing binary models can be found that fit the available photometric data, these models would produce obviously composite spectroscopic cross-correlation functions ( $\mathrm{CCFs}$ ) that are inconsistent with the observed CCFs. For example, in all cases the spectral line bisector spans (BSs) computed from the simulated CCFs have scatter in excess of $900 \mathrm{~m} \mathrm{~s}^{-1}$, with a maximum simulated value of $4.54 \mathrm{~km} \mathrm{~s}^{-1}$, whereas the scatter of the measured FEROS BSs is $\sim 100 \mathrm{~m} \mathrm{~s}^{-1}$. Similarly the RVs of the simulated CCFs are in excess of $500 \mathrm{~m} \mathrm{~s}^{-1}$, whereas the observed FEROS RVs have a scatter of $130 \mathrm{~m} \mathrm{~s}^{-1}$ (dominated by the planetary signals). We conclude that the transiting signals are indeed due to a planet, and HATS-59 is not a blended stellar eclipsing binary.

\subsection{Global Modeling of the Data}

To measure the orbital and physical properties of the planets, we modeled all the photometric data (the HATSouth and follow-up photometric data) and the high-precision RV measurements following Pál et al. (2008), Bakos et al. (2010) and Hartman et al. (2012).

All the photometric light curves were modeled using the Mandel \& Agol (2002) transit models with fixed quadratic limb-darkening coefficients taken from Claret (2004). For the HATSouth discovery photometric light curves, we also considered a dilution factor for the transit depth that accounts for possible blends from neighboring stars and possible overcorrection introduced by the trend filtering algorithm (TFA; removes trends shared with other stars; Bakos et al. 2010; Kovács et al. 2005). As for the photometric follow-up light curves, the systematic trends were corrected by including a quadratic trend to the transit model. We also added a linear trend, with up to three parameters, to reconstruct the shape of the PSF. This trend compensates for changes in the PSF during the observations, which could be due to poor guiding, nonphotometric conditions, or changes in the seeing during the transit observations.

We fit the RVs, taken with different spectrographs, with a Keplerian orbit allowing the zero-point and the RV jitter, for each instrument, to vary independently in the fit. This ensures that the best-fitting model is self-consistent with the data set. Our RVs support the existence of a second planet on top of the transiting one, and therefore models with two planets were considered in the modeling. We considered four different scenarios where one or both of the planets had a fixed circular orbit, or was allowed to have non-zero eccentricity. To choose between the different scenarios, we estimated the Bayesian evidence for each model following Weinberg et al. (2013), and 

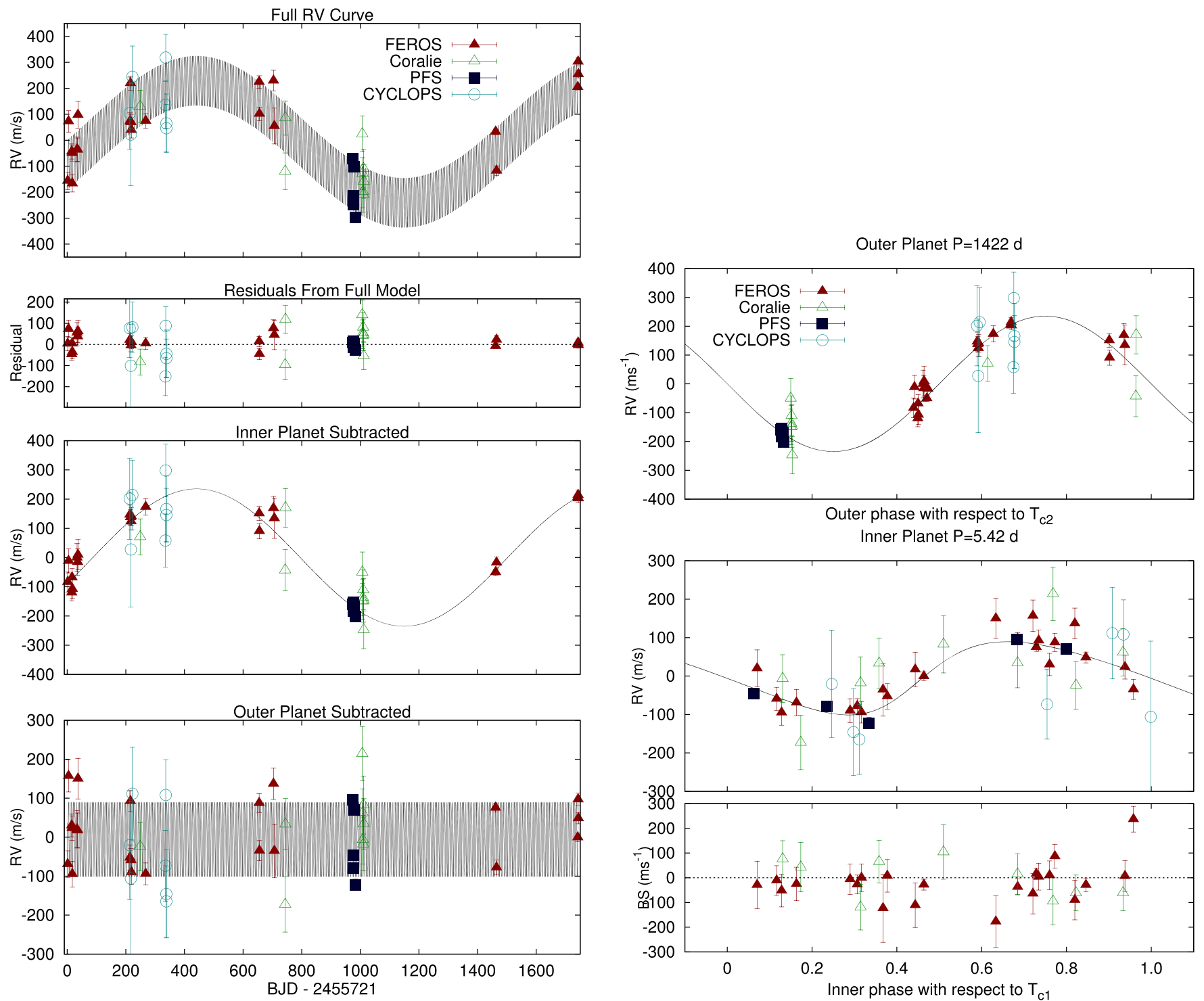

Figure 5. Top left: FEROS, Coralie, PFS, and CYCLOPS high-precision RV measurements, together with our best-fit two-planet orbit model, plotted as a function of time. The center-of-mass velocity has been subtracted. The error bars include the jitter which is varied independently for each instrument in the fit. Left, second panel: $\mathrm{RV} O-C$ residuals from the two-planet model, plotted as a function of time. Left, third panel: RV residuals after subtracting only the model variation due to the inner planet, plotted as a function of time. Left, bottom panel: RV residuals after subtracting only the model variation due to the outer planet, plotted as a function of time. Top right: RV residuals after subtracting only the model variation due to the inner planet, plotted as a function of phase of the outer planet. Here zero phase is the time of inferior conjunction for the outer planet. Right, second panel: RV residuals after subtracting only the model variation due to the outer planet, plotted as a function of phase of the inner planet. Right, bottom panel: Spectral line bisector spans (BSs) plotted as a function of phase of the inner planet. Note the different vertical scales of all of the panels.

then adopted the model with the highest evidence, which we find to be a model in which the interior transiting planet has a non-zero eccentricity, while the exterior planet has a circular orbit. The evidence for this model is a modest factor of 2.4 times greater than the evidence for the model in which both planets are assumed to have circular orbits, 7 times greater than the model in which the interior planet is circular and the exterior planet has an eccentric orbit, and 19 times greater than the model in which both planets have non-zero eccentricities.

The posterior distributions for each parameter and hence the median parameters along with their $1 \sigma$ uncertainties were estimated using the differential evolution Markov Chain Monte Carlo procedure (DEMCMC; ter Braak 2006) and are provided in Table 4. We find that the transiting planet HATS-59b has a mass of $0.806 \pm 0.069 M_{\mathrm{J}}$, a radius of $1.126 \pm 0.077 R_{\mathrm{J}}$, and a non-zero eccentricity of $e=0.129 \pm 0.049$. For the second planet, which we dub HATS-59c, we find that is well fit by a circular Keplerian orbit with $P=1422 \pm 14$ days, $K=$ $224 \pm 14 \mathrm{~m} \mathrm{~s}^{-1}$, implying a minimum mass for the companion of $m \sin i=12.70 \pm 0.87 M_{\mathrm{J}}$, where $i$ is the orbital inclination of HATS-59c.

\section{Discussion}

We present the discovery of HATS-59, the first multi-planet system detected by the HATSouth survey. The inner planet, HATS-59b, is a transiting hot Jupiter on an eccentric orbit, completing one revolution every $\approx 5$ days. The outer planet, HATS-59c, is a cold massive giant planet on a circular orbit with a period of 1422 days. We note the $m \sin i$ for HATS-59b, $\mathrm{c}$ is very close to the theoretical limit for deuterium burning for a solar metallicity object, and thus it may be a very low mass 


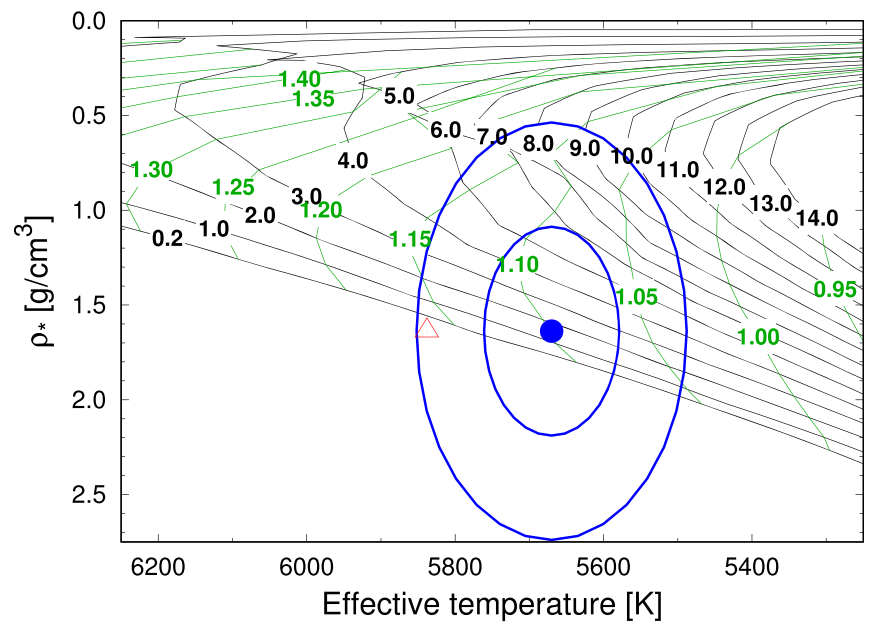

Figure 6. Model isochrones (black solid lines) from Yi et al. (2001) for the measured metallicity of HATS-59. The age of each isochrone in Gyr is labeled in black font. We also show evolutionary tracks for stars of fixed mass (dashed green lines) with the mass of each tracked labeled in solar mass units in green font. The adopted values of $T_{\mathrm{eff} \star}$ and $\rho_{\star}$ are shown using the filled blue circle together with their $1 \sigma$ and $2 \sigma$ confidence ellipsoids (blue lines). The initial values of $T_{\text {eff } \star}$ and $\rho_{\star}$ from the first ZASPE and light curve analysis are represented with the red open triangle.

brown dwarf rather than a giant exoplanet, although the distinction is unlikely to change the physical characteristics of the object.

\subsection{Possible Formation Scenarios of HATS-59b,c}

The architecture of HATS-59b,c poses a challenge for planet formation and migration scenarios. Can core accretion explain the presence of a hot Jupiter and a massive gas giant in the same system? Schlaufman (2018) found that planets with $M>10 M_{\mathrm{J}}$ do not preferentially orbit metal-rich solar-like stars, suggesting that these objects most likely did not form via core accretion but via gravitational instability. The architecture of HATS-59b,c hence suggests that both core accretion and gravitational instability could have occurred in the same system, which was also previously suggested by Triaud et al. (2017) for WASP-53bc and WASP-81bc.

The current water iceline is around $2.92 \mathrm{au}$, suggesting that both HATS-59b and HATS-59c formed beyond the iceline and then migrated inwards to their present locations. The presence of HATS-59c, a massive companion close to the deuterium burning limit (Mollière \& Mordasini 2012), could have scattered HATS-59b inwards resulting in its present eccentric orbit. Due to its mass, type-II migration is reduced even below the viscous limit for HATS-59c (Baruteau et al. 2014), resulting in only little inward migration, potentially explaining its long period.

\subsection{Transit Timing Variations}

Variations in the times of transits can be attributed to the presence of a secondary planet in the system (e.g., Agol et al. 2005; Mancini et al. 2016; Almenara et al. 2018). The maximum transit variation expected for the inner planet is on the order of $10^{-10} \mathrm{~s}$, undetectable with current instruments. However, this depends on the mutual inclination between the inner and outer planet.
Table 3

Stellar Parameters for HATS-59

\begin{tabular}{|c|c|c|}
\hline Parameter & Value & Source \\
\hline \multicolumn{3}{|l|}{ Identifying Information } \\
\hline R.A. (h:m:s) & $11^{\mathrm{h}} 21^{\mathrm{m}} 18^{\mathrm{s}} .00$ & 2MASS \\
\hline Decl. (d:m:s) & $-22^{\circ} 23^{\prime} 17^{\prime \prime} 4$ & 2MASS \\
\hline R.A.p.m. $\left(\mathrm{mas} \mathrm{yr}^{-1}\right)$ & $-24.16 \pm 0.047$ & Gaia DR2 \\
\hline Decl.p.m. $\left(\operatorname{mas~yr}^{-1}\right)$ & $0.92 \pm 0.03$ & Gaia DR2 \\
\hline Parallax (mas) & $1.52 \pm 0.03$ & Gaia DR2 \\
\hline GSC ID & GSC 6090-00133 & GSC \\
\hline 2MASS ID & $\begin{array}{c}\text { 2MASS } 11211786- \\
2223174\end{array}$ & 2MASS \\
\hline \multicolumn{3}{|l|}{ Spectroscopic properties } \\
\hline$T_{\mathrm{eff} \star}(\mathrm{K})$ & $5670 \pm 91$ & ZASPE $^{\mathrm{a}}$ \\
\hline$[\mathrm{Fe} / \mathrm{H}]$ & $0.180 \pm 0.064$ & ZASPE \\
\hline$v \sin i\left(\mathrm{~km} \mathrm{~s}^{-1}\right)$ & $2.80 \pm 0.61$ & ZASPE \\
\hline$\gamma_{\mathrm{RV}}\left(\mathrm{m} \mathrm{s}^{-1}\right)$ & $-10887 \pm 11$ & FEROS \\
\hline \multicolumn{3}{|l|}{ Photometric properties } \\
\hline$B$ (mag) & $14.727 \pm 0.020$ & APASS \\
\hline$V$ (mag) & $13.951 \pm 0.030$ & APASS \\
\hline$g(\mathrm{mag})$ & $14.286 \pm 0.030$ & APASS \\
\hline$r(\mathrm{mag})$ & $13.725 \pm 0.030$ & APASS \\
\hline$i$ (mag) & $13.551 \pm 0.040$ & APASS \\
\hline$J$ (mag) & $12.590 \pm 0.024$ & 2MASS \\
\hline$H(\mathrm{mag})$ & $12.299 \pm 0.030$ & 2MASS \\
\hline$K_{s}(\mathrm{mag})$ & $12.238 \pm 0.030$ & 2MASS \\
\hline$G(\mathrm{mag})$ & 13.785 & Gaia DR2 \\
\hline \multicolumn{3}{|l|}{ Derived properties } \\
\hline$M_{\star}\left(M_{\odot}\right)$ & $1.038 \pm 0.039$ & $\mathrm{Y}^{2}+\rho_{\star}+\mathrm{ZASPE}^{\mathrm{b}}$ \\
\hline$R_{\star}\left(R_{\odot}\right)$ & $1.036 \pm 0.067$ & $\mathrm{Y}^{2}+\rho_{\star}+\mathrm{ZASPE}$ \\
\hline $\log g_{\star}(\operatorname{cgs})$ & $4.422 \pm 0.053$ & $\mathrm{Y}^{2}+\rho_{\star}+\mathrm{ZASPE}$ \\
\hline$\rho_{\star}\left(\mathrm{g} \mathrm{cm}^{-3}\right)^{\mathrm{c}}$ & $1.59 \pm 0.54$ & Light curves \\
\hline$\rho_{\star}\left(\mathrm{g} \mathrm{cm}^{-3}\right)^{\mathrm{c}}$ & $1.31 \pm 0.24$ & $\begin{array}{c}\mathrm{Y}^{2}+\text { Light curves } \\
+ \text { ZASPE }\end{array}$ \\
\hline$L_{\star}\left(L_{\odot}\right)$ & $0.99 \pm 0.16$ & $\mathrm{Y}^{2}+\rho_{\star}+\mathrm{ZASPE}$ \\
\hline$M_{V}(\mathrm{mag})$ & $4.86 \pm 0.19$ & $\mathrm{Y}^{2}+\rho_{\star}+\mathrm{ZASPE}$ \\
\hline$M_{K}(\mathrm{mag}, \mathrm{ESO})$ & $3.24 \pm 0.14$ & $\mathrm{Y}^{2}+\rho_{\star}+\mathrm{ZASPE}$ \\
\hline Age $(\mathrm{Gyr})$ & $4.3 \pm 2.3$ & $\mathrm{Y}^{2}+\rho_{\star}+\mathrm{ZASPE}$ \\
\hline$A_{V}(\mathrm{mag})^{\mathrm{d}}$ & $0.091 \pm 0.074$ & $\mathrm{Y}^{2}+\rho_{\star}+\mathrm{ZASPE}$ \\
\hline Distance (pc) & $654 \pm 14$ & Gaia DR2 \\
\hline
\end{tabular}

Notes.

a ZASPE = "Zonal Atmospherical Stellar Parameter Estimator" method for the analysis of the FEROS high-resolution spectra (Brahm et al. 2017b). Similar to previous works, these parameters rely primarily on ZASPE, but they also have a small dependence on the iterative analysis of the isochrone search and global modeling of the data. For more information see the text.

${ }^{\mathrm{b}} \mathrm{Y}^{2}$ Isochrones (Yi et al. 2001) $+\rho_{\star}+\mathrm{ZASPE}=$ the stellar density which is used as a luminosity indicator, and the ZASPE results.

c Two different values for $\rho_{\star}$ are listed. The first is determined from the global fit to the light curves and RV data, without imposing a constraint that the parameters match the stellar evolution models. The second value results from restricting the posterior distribution to combinations of $\rho_{\star}+T_{\mathrm{eff} \star}+[\mathrm{Fe} / \mathrm{H}]$ that match to a $\mathrm{Y}^{2}$ stellar model.

d The star extinction in the $V$ band determined by comparing the expected magnitude from Isochrones $+\rho_{\star}+$ ZASPE model for the star to the catalog broad-band photometry listed in the table. The extinction law is from Cardelli et al. (1989).

\subsection{The Inner Transiting Planet HATS-59b}

In Figure 7, we plot the masses and radii of all the transiting exoplanets having these parameters measured with a precision better than $20 \%$. HATS-59b lies in a densely populated region of the parameter space, where numerous non inflated giant 


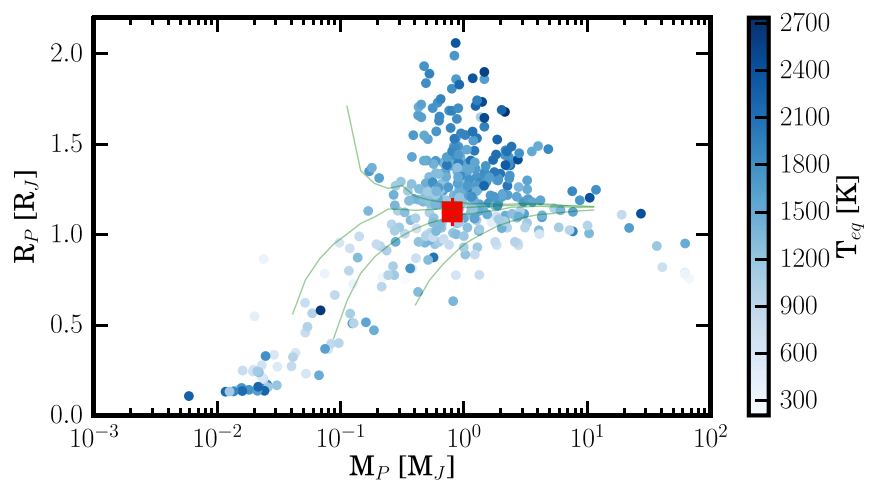

Figure 7. Mass-radius diagram for the full population of well characterized transiting planets color coded by their equilibrium temperature. HATS-59b is shown in red. The Fortney et al. (2007) models of planetary structure are also plotted as green lines. The four models correspond to gaseous planets with $a=0.045 \mathrm{au}$, age $=4.3 \mathrm{Gyr}$, and core masses of $0,25,50$, and $100 M_{\oplus}$.

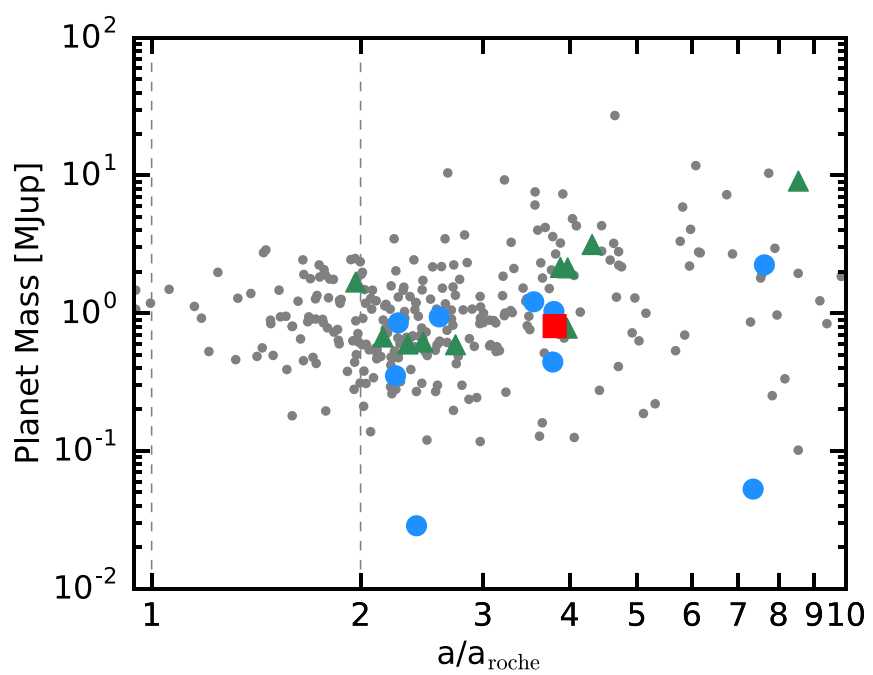

Figure 8. Planetary mass vs. $a / a_{\text {roche }}$ for single (small gray circles), known multi-planetary systems (blue circles), and systems showing a linear trend (green triangles). HATS-59b is shown as a red square. Most of the multiplanetary systems have $a / a_{\text {roche }}>2$, which supports the high eccentricity migration scenario.

planets with similar properties have been detected. In terms of structure, HATS-59b is similar to HAT-P-29 b $\left(M_{p}=0.78 M_{\mathrm{J}}\right.$, $R_{p}=1.11 R_{\mathrm{J}}$, and $P=5.7$ days; Buchhave et al. 2011); and $\mathrm{K} 2-115 \mathrm{~b},\left(M_{p}=0.84 M_{\mathrm{J}}, R_{p}=1.12 R_{\mathrm{J}}\right.$, and $P=20.3$ days; Shporer et al. 2017), however with a significantly shorter period.

We compare the mass and radius of HATS-59b to the theoretical models of Fortney et al. (2007), for a hydrogenhelium dominated planets with different core masses, at a distance of $0.045 \mathrm{au}$, and an age of $4.3 \mathrm{Gyr}$. We find that its composition is consistent with a gas-dominated planet with a core mass $M_{\mathrm{c}}<25 M_{\oplus}$. However, these models assume that all the solid material is located inside the core. According to Thorngren et al. (2016), HATS-59b could have a larger amount of heavy elements in its interior $\left(\sim 50 M_{\oplus}\right)$ if they are predominantly mixed in the gaseous envelope.

\subsubsection{Possible Migration Scenarios of HATS-59b}

Hot Jupiters are thought to form beyond the iceline and migrate inwards via disk or high eccentricity migration, where the latter requires an outer planetary or stellar companion.

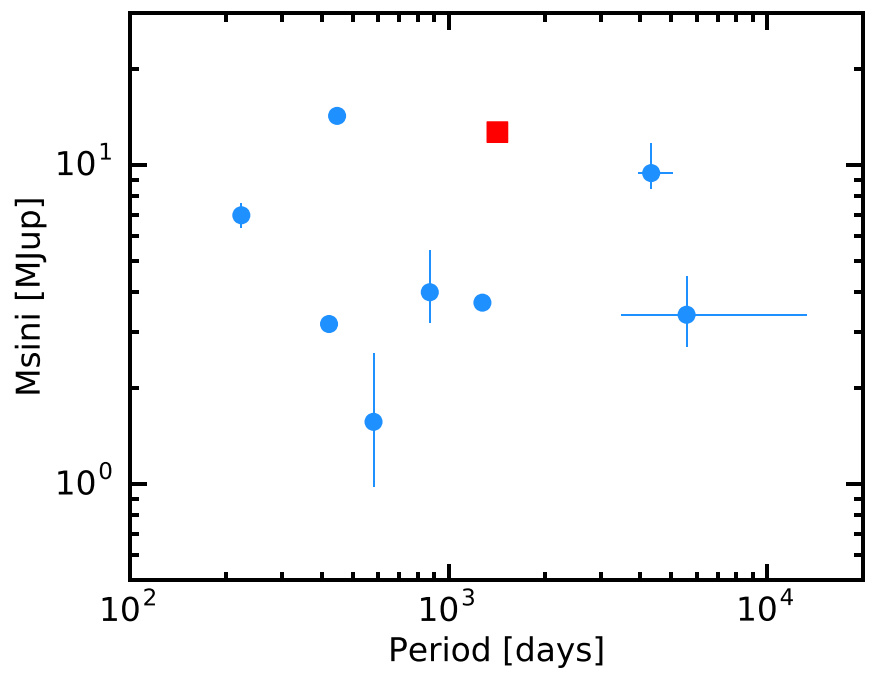

Figure 9. Msini vs. period for the outer companions where the orbit was fully observed (blue circle). HATS-59c (red square) has the third longest period, where only nine companions have been characterized.

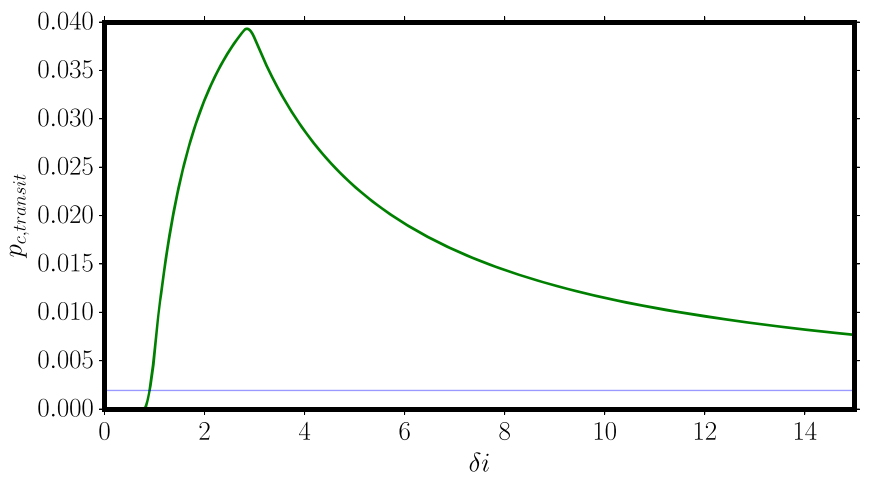

Figure 10. Transit probability for HATS-59c for an aligned configuration with HATS-59b as a function of the maximum separation in inclination between both planets. The blue line shows the a priori probability for HATS-59c to transit. A maximum probability of $\approx 4 \%$ would occur if the orbital plane of HATS-59c is inclined around $3 \mathrm{deg}$ with respect to that of HATS-59b.

Observations of the projected spin-orbit angle via the RossiterMcLaughin (RM) effect provides an approach to distinguish between these migration scenarios. Disk migration predicts circular and aligned orbits, whereas the high eccentricity migration can produce a broad range of obliquities, depending mostly on the scattering mechanism and on the effectiveness of tidal interactions at damping obliquities.

The amplitude of the RM effect scales with $v \sin i$, the projected rotational velocity of the star. We predict an $\mathrm{RM}$ amplitude of $23-36 \mathrm{~m} \mathrm{~s}^{-1}$ for $v \sin i=2.2-3.4 \mathrm{~km} \mathrm{~s}^{-1}$. Measuring the RM amplitude for this faint $\operatorname{star}(V=13.951 \pm$ $0.030 \mathrm{mag}$ ), is challenging but plausible using HIRES (Vogt et al. 1994; Wang et al. 2018) on the Keck telescope or with the new high-resolution spectrograph, ESPRESSO (Pepe et al. 2014) at the Very Large Telescope.

Disk migration predicts that planets can migrate up until they reach the planet-star Roche separation $\left(a_{\text {roche }}\right)$, the critical distance within which a planet would start losing mass (Faber et al. 2005). On the other hand, high-eccentricity migration predicts planets will circularize at a semimajor axis greater than $2 a_{\text {roche }}$. This mechanism would require that hot Jupiters are excited to eccentric orbits, often by being scattered by a distant 
Table 4

Parameters for the Planets HATS-59b,c

\begin{tabular}{|c|c|c|}
\hline Parameter & $\begin{array}{l}\text { HATS-59b } \\
\text { Value }^{\mathrm{a}}\end{array}$ & $\begin{array}{r}\text { HATS-59c } \\
\text { Value }^{\mathrm{a}}\end{array}$ \\
\hline \multicolumn{3}{|l|}{ Light curve parameters } \\
\hline$P$ (days) & $5.416081 \pm 0.000016$ & $1422 \pm 14$ \\
\hline$T_{c}(\mathrm{BJD})^{\mathrm{b}}$ & $2456620.66527 \pm 0.00052$ & $2456521 \pm 11$ \\
\hline$T_{14}(\text { days })^{\mathrm{b}}$ & $0.1497 \pm 0.0017$ & $0.957 \pm 0.054$ \\
\hline$T_{12}=T_{34}(\text { days })^{\mathrm{b}}$ & $0.0186 \pm 0.0016$ & $0.0863 \pm 0.0011$ \\
\hline$a / R_{\star}$ & $12.66 \pm 0.77$ & $518 \pm 32$ \\
\hline$\zeta / R_{\star}{ }^{\mathrm{c}}$ & $15.23 \pm 0.13$ & $\ldots$ \\
\hline$R_{p} / R_{\star}$ & $0.1116 \pm 0.0021$ & $\cdots$ \\
\hline$b^{2}$ & $0.209_{-0.056}^{+0.054}$ & $\ldots$ \\
\hline$b \equiv a \cos i / R_{\star}$ & $0.457_{-0.066}^{+0.056}$ & $\cdots$ \\
\hline$i(\mathrm{deg})$ & $88.10 \pm 0.33$ & $\cdots$ \\
\hline \multicolumn{3}{|c|}{ Limb-darkening coefficients ${ }^{\mathrm{d}}$} \\
\hline$c_{1}, g$ (linear term) & 0.5965 & $\cdots$ \\
\hline $\begin{array}{l}c_{2}, g \text { (quad- } \\
\text { ratic term) }\end{array}$ & 0.2045 & $\cdots$ \\
\hline$c_{1}, R$ & 0.3628 & $\cdots$ \\
\hline$c_{2}, R$ & 0.3129 & $\cdots$ \\
\hline$c_{1}, r$ & 0.3896 & $\cdots$ \\
\hline$c_{2}, r$ & 0.3085 & $\cdots$ \\
\hline$c_{1}, i$ & 0.2930 & $\cdots$ \\
\hline$c_{2}, i$ & 0.3208 & $\cdots$ \\
\hline$c_{1}, z$ & 0.2259 & $\cdots$ \\
\hline$c_{2}, z$ & 0.3232 & $\cdots$ \\
\hline \multicolumn{3}{|l|}{ RV parameters } \\
\hline$K\left(\mathrm{~m} \mathrm{~s}^{-1}\right)$ & $92.1 \pm 7.8$ & $224 \pm 14$ \\
\hline$e^{\mathrm{e}}$ & $0.129 \pm 0.049$ & $<0.083$ \\
\hline$\omega$ & $227 \pm 29$ & $\ldots$ \\
\hline$\sqrt{e} \cos \omega$ & $-0.233 \pm 0.084$ & $\cdots$ \\
\hline$\sqrt{e} \sin \omega$ & $-0.25_{-0.11}^{+0.18}$ & $\cdots$ \\
\hline$e \cos \omega$ & $-0.082 \pm 0.034$ & $\cdots$ \\
\hline$e \sin \omega$ & $-0.090 \pm 0.065$ & $\cdots$ \\
\hline $\begin{array}{l}\text { FEROS RV jit- } \\
\text { ter }\left(\mathrm{m} \mathrm{s}^{-1}\right)^{\mathrm{f}}\end{array}$ & $<20.7$ & $\cdots$ \\
\hline $\begin{array}{l}\text { Coralie RV jit- } \\
\text { ter }\left(\mathrm{m} \mathrm{s}^{-1}\right)^{\mathrm{f}}\end{array}$ & $58 \pm 44$ & $\cdots$ \\
\hline $\begin{array}{l}\text { PFS RV jit- } \\
\text { ter }\left(\mathrm{m} \mathrm{s}^{-1}\right)^{\mathrm{f}}\end{array}$ & $24 \pm 14$ & $\cdots$ \\
\hline $\begin{array}{l}\text { CYCLOPS RV jit- } \\
\text { ter }\left(\mathrm{m} \mathrm{s}^{-1}\right)^{\mathrm{f}}\end{array}$ & $93 \pm 40$ & $\cdots$ \\
\hline \multicolumn{3}{|l|}{ Planetary parameters } \\
\hline$M_{p}\left(M_{\mathrm{J}}\right)$ & $0.806 \pm 0.069$ & $\ldots$ \\
\hline$M_{p} \sin i\left(M_{\mathrm{J}}\right)$ & $\ldots$ & $12.70 \pm 0.87$ \\
\hline$R_{p}\left(R_{\mathrm{J}}\right)$ & $1.126 \pm 0.077$ & $\ldots$ \\
\hline$C\left(M_{p}, R_{p}\right)^{\mathrm{g}}$ & 0.05 & $\cdots$ \\
\hline$\rho_{p}\left(\mathrm{~g} \mathrm{~cm}^{-3}\right)$ & $0.70 \pm 0.16$ & $\cdots$ \\
\hline $\log g_{p}(\mathrm{cgs})$ & $3.195 \pm 0.069$ & $\cdots$ \\
\hline$a(\mathrm{au})$ & $0.06112 \pm 0.00076$ & $2.504 \pm 0.035$ \\
\hline$T_{\mathrm{eq}}(\mathrm{K})^{\mathrm{h}}$ & $1128 \pm 40$ & $175.9 \pm 6.4$ \\
\hline
\end{tabular}

massive companion, and survived the tidal dissipation process required to circularize their final orbits (Faber et al. 2005; Ford 2006).

Many distant planetary companions to hot Jupiters have been detected (Knutson et al. 2014). In Figure 8, we show planetary mass plotted against $a / a_{\text {roche }}$, where

$$
a_{\text {roche }}=2.7 R_{p}\left(\frac{M_{*}}{M_{p}}\right)^{1 / 3},
$$

Table 4

(Continued)

\begin{tabular}{lcc}
\hline \hline Parameter & $\begin{array}{r}\text { HATS-59b } \\
\text { Value }^{\mathrm{a}}\end{array}$ & $\begin{array}{r}\text { HATS-59c } \\
\text { Value }^{\mathrm{a}}\end{array}$ \\
\hline$\Theta^{\mathrm{i}}$ & $0.0841 \pm 0.0093$ & $\ldots$ \\
$\langle F\rangle\left(\mathrm{erg} \mathrm{s}^{-1} \mathrm{~cm}^{-2}\right)^{\mathrm{j}}$ & $(3.66 \pm 0.53) \times 10^{8}$ & $(2.16 \pm 0.32) \times 10^{5}$ \\
\hline
\end{tabular}

Notes.

${ }^{\mathrm{a}}$ We provide the median value and the $68.3 \%(1 \sigma)$ confidence intervals for all the parameters. Reported results assume an eccentric orbit for HATS-59b and a circular orbit for HATS-59c.

${ }^{\mathrm{b}}$ Reported times are in Barycentric Julian Date calculated directly from UTC, without correction for leap seconds. $T_{c}$ : Reference epoch of mid transit that minimizes the correlation with the orbital period. Note that HATS-59c has not been observed to transit. We list here the time of mid transit, implied by the orbital solution, in the event that the orbital inclination permits transits. $T_{14}$ : total transit duration, time between first to last contact; $T_{12}=T_{34}$ : ingress/ egress time, time between first and second, or third and fourth contact. For HATS-59c $T_{14}$ and $T_{12}$ are calculated assuming central transits $\left(i=90^{\circ}\right.$ orbit) and a Jupiter radius for the planet.

${ }^{\mathrm{c}}$ Reciprocal of the half duration of the transit used as a jump parameter in our MCMC analysis in place of $a / R_{\star}$. It is related to $a / R_{\star}$ by the expression $\zeta / R_{\star}=a / R_{\star}(2 \pi(1+e \sin \omega)) /\left(P \sqrt{1-b^{2}} \sqrt{1-e^{2}}\right)$ (Bakos et al. 2010).

${ }^{\mathrm{d}}$ Values for a quadratic law, adopted from the tabulations by Claret (2004) according to the spectroscopic (ZASPE) parameters listed in Table 3.

${ }^{\mathrm{e}}$ For HATS-59c, we list the $95 \%$ confidence upper-limit on the eccentricity. All the other parameters are estimated assuming a circular orbit for this planet.

${ }^{\mathrm{f}}$ Astrophysical or instrumental error added in quadrature to the original RV errors. This term is varied in the fit independently for each instrument assuming a prior that is inversely proportional to the jitter.

${ }^{\mathrm{g}}$ Correlation coefficient between the planetary mass $M_{p}$ and radius $R_{p}$ determined from the parameter posterior distribution via $C\left(M_{p}, R_{p}\right)=$ $\left.\left\langle\left(M_{p}-\left\langle M_{p}\right\rangle\right)\left(R_{p}-\left\langle R_{p}\right\rangle\right)\right\rangle /\left(\sigma_{M_{p}} \sigma_{R_{p}}\right)\right\rangle$, where $\langle\cdot\rangle$ is the expectation value, and $\sigma_{x}$ is the std. dev. of $x$.

${ }^{\mathrm{h}}$ Planet equilibrium temperature averaged over the orbit, calculated assuming a Bond albedo of zero, and that flux is reradiated from the full planet surface.

${ }^{\mathrm{i}}$ The Safronov number is given by $\Theta=\frac{1}{2}\left(V_{\text {esc }} / V_{\text {orb }}\right)^{2}=\left(a / R_{p}\right)\left(M_{p} / M_{\star}\right)$ (see Hansen \& Barman 2007)

${ }^{\mathrm{j}}$ Incoming flux per unit surface area, averaged over the orbit.

Table 5

Future Transit Windows

\begin{tabular}{cc}
\hline \hline $\begin{array}{c}\text { Date } \\
(\mathrm{UT})\end{array}$ & $\begin{array}{c}\text { Sun RA distance } \\
(\mathrm{hr})\end{array}$ \\
\hline 2021 May 30 & 6.8 \\
2025 Apr 21 & 9.4 \\
2029 Mar 13 & 12.2 \\
\hline
\end{tabular}

for all hot Jupiters whose mass and radii are determined with a precision better than $30 \%$ (small gray circles). Blue circles show all the hot Jupiters with a fully resolved orbit of the outer planetary companion and green triangle represent the systems whose RVs show a linear trend, taken from Knutson et al. (2014). The position of HATS-59b is shown with a red square. All but one multi-planet system have $a / a_{\text {roche }}>2$, HAT-P-7b, with a value $a / a_{\text {roche }}$ only slightly lower than 2 . The available data on hot Jupiters with companions indicate that high eccentricity migration could be the main mechanism for placing the gas giant on a close-in orbit in these systems.

We compare the parameters of HATS-59c to all the detected planetary companions whose orbit is fully resolved. Figure 9 
shows the position of HATS-59c (red square) on the minimum mass-period diagram with the other discovered companions (blue circles). With a period of 1422 days, HATS-59c has the third longest period, indicating how few outer companions to transiting hot Jupiters have been characterized due to the lack of RV follow-up observations. All of the companions have minimum masses above $1 M_{\mathrm{J}}$, which is most likely due to selection effects with a detection limit of $\sim 20 \mathrm{~m} \mathrm{~s}^{-1}$ for a planet orbiting a Sun analog.

\subsection{Possible Transits of HATS-59c}

As was stated in the previous section, knowing the mutual inclination between HATS-59b and HATS-59c can be useful to further clarify the possible migration path of this system. The host star is too faint for the GAIA mission to be able to measure the astrometric signal of HATS-59c. However, the inclination of HATS-59c with respect to the plane of the sky could be measured if it also transits its star. While the a priori probability of transit for HATS-59c is $\sim 0.2 \%$, if we consider that the two planets are co-planar, then the probability of transit raises by one order of magnitude. Figure 10 shows the transit probability of HATS-59c for different assumed maximum mutual inclinations $\left(\delta_{i}\right)$ between the orbital plane of the planets. The probabilities were computed following the formalism of Beatty $\&$ Seager (2010). The maximum probability $(3.8 \%)$ occurs if the mutual inclination between the planets is around $3 \mathrm{deg}$.

The future transit windows for HATS-59c are listed in Table 5. In this table, we indicate the center of the transit window and the distance of the target from the Sun at the time of putative transit center. Currently, the width of the transit window is quite large ( $>50$ days) due to the large uncertainties in the ephemeris. Longterm RV monitoring of the system would be useful to further constrain the width of the transit window.

Development of the HATSouth project was funded by NSF MRI grant NSF/AST-0723074, operations have been supported by NASA grants NNX09AB29G, NNX12AH91H, and NNX17AB61G, and follow-up observations receive partial support from grant NSF/AST-1108686. P.S. would like to thank Bertram Bitsch for useful discussions. A.J. acknowledges support from FONDECYT project 1171208, BASAL CATA PFB-06, and project IC120009 "Millennium Institute of Astrophysics (MAS)" of the Millenium Science Initiative, Chilean Ministry of Economy. R.B. acknowledges support from project IC120009 "Millenium Institute of Astrophysics (MAS)" of the Millennium Science Initiative, Chilean Ministry of Economy. L.M. acknowledges support from the Italian Minister of Instruction, University and Research (MIUR) through FFABR 2017 fund. J.H. acknowledges support from NASA grant NNX14AE87G. V.S. acknowledges support form BASAL CATA PFB-06. A.V. is supported by the NSF Graduate Research Fellowship, Grant No. DGE 1144152. This work has made use of data from the European Space Agency (ESA) mission Gaia (https://www. cosmos.esa.int/gaia), processed by the Gaia Data Processing and Analysis Consortium (DPAC, https://www.cosmos.esa. int/web/gaia/dpac/consortium). Funding for the DPAC has been provided by national institutions, in particular the institutions participating in the Gaia Multilateral Agreement. This work is based on observations made with ESO Telescopes at the La Silla Observatory. This paper also uses observations obtained with facilities of the Las Cumbres
Observatory Global Telescope. We acknowledge the use of the AAVSO Photometric All-Sky Survey (APASS), funded by the Robert Martin Ayers Sciences Fund, and the SIMBAD database, operated at CDS, Strasbourg, France. Operations at the MPG $2.2 \mathrm{~m}$ Telescope are jointly performed by the Max Planck Gesellschaft and the European Southern Observatory. The imaging system GROND has been built by the highenergy group of MPE in collaboration with the LSW Tautenburg and ESO. We thank the MPG $2.2 \mathrm{~m}$ telescope support team for their technical assistance during observations.

\section{ORCID iDs}

P. Sarkis (iD https://orcid.org/0000-0001-8128-3126 J. D. Hartman (ib https://orcid.org/0000-0001-8732-6166 G. Á. Bakos (iD https://orcid.org/0000-0001-7204-6727

R. Brahm (i) https://orcid.org/0000-0002-9158-7315

D. Bayliss (iD https://orcid.org/0000-0001-6023-1335 L. Mancini (i) https://orcid.org/0000-0002-9428-8732 W. Bhatti (iD https://orcid.org/0000-0002-0628-0088

K. Penev (iD https://orcid.org/0000-0003-4464-1371

P. Arriagada (i) https://orcid.org/0000-0002-3578-551X

R. P. Butler (10 https://orcid.org/0000-0003-1305-3761

J. D. Crane (iD https://orcid.org/0000-0002-5226-787X

C. G. Tinney (1) https://orcid.org/0000-0002-7595-0970

S. Durkan (iD https://orcid.org/0000-0002-3663-3251

V. Suc (i) https://orcid.org/0000-0001-7070-3842

L. A. Buchhave (1) https://orcid.org/0000-0003-1605-5666

\section{References}

Agol, E., Steffen, J., Sari, R., \& Clarkson, W. 2005, MNRAS, 359, 567 Almenara, J. M., Díaz, R. F., Hébrard, G., et al. 2018, A\&A, 615, A90 Artymowicz, P. 1993, ApJ, 419, 166

Bakos, G. Á, Csubry, Z., Penev, K., et al. 2013, PASP, 125, 154

Bakos, G. Á, Howard, A. W., Noyes, R. W., et al. 2009, ApJ, 707, 446

Bakos, G. Á, Torres, G., Pál, A., et al. 2010, ApJ, 710, 1724

Baruteau, C., Crida, A., Paardekooper, S.-J., et al. 2014, in Protostars and Planets VI, ed. H. Beuther et al. (Tucson, AZ: Univ. Arizona Press), 667 Batygin, K., Bodenheimer, P., \& Laughlin, G. 2009, ApJL, 704, L49

Bayliss, D., Zhou, G., Penev, K., et al. 2013, AJ, 146, 113

Beatty, T. G., \& Seager, S. 2010, ApJ, 712, 1433

Becker, J. C., Vanderburg, A., Adams, F. C., Rappaport, S. A., \& Schwengeler, H. M. 2015, ApJL, 812, L18

Brahm, R., Jordán, A., \& Espinoza, N. 2017a, PASP, 129, 034002

Brahm, R., Jordán, A., Hartman, J., \& Bakos, G. 2017b, MNRAS, 467, 971 Brown, T. M., Baliber, N., Bianco, F. B., et al. 2013, PASP, 125, 1031 Buchhave, L. A., Bakos, G. Á, Hartman, J. D., et al. 2010, ApJ, 720, 1118 Buchhave, L. A., Bakos, G. Á, Hartman, J. D., et al. 2011, ApJ, 733, 116 Buhler, P. B., Knutson, H. A., Batygin, K., et al. 2016, ApJ, 821, 26 Butler, R. P., Marcy, G. W., Williams, E., et al. 1996, PASP, 108, 500 Cardelli, J. A., Clayton, G. C., \& Mathis, J. S. 1989, ApJ, 345, 245 Claret, A. 2004, A\&A, 428, 1001

Crane, J. D., Shectman, S. A., Butler, R. P., et al. 2010, Proc. SPIE, 7735 773553

Dopita, M., Hart, J., McGregor, P., et al. 2007, Ap\&SS, 310, 255

Endl, M., Caldwell, D. A., Barclay, T., et al. 2014, ApJ, 795, 151 Espinoza, N., Bayliss, D., Hartman, J. D., et al. 2016, AJ, 152, 108 Faber, J. A., Rasio, F. A., \& Willems, B. 2005, Icar, 175, 248 Ford, E. B. 2006, ApJ, 642, 505

Fortney, J. J., Marley, M. S., \& Barnes, J. W. 2007, ApJ, 659, 1661 Goldreich, P., \& Tremaine, S. 1980, ApJ, 241, 425

Greiner, J., Bornemann, W., Clemens, C., et al. 2008, PASP, 120, 405 Hansen, B. M. S., \& Barman, T. 2007, ApJ, 671, 861

Hardy, R. A., Harrington, J., Hardin, M. R., et al. 2017, ApJ, 836, 143

Hartman, J. D., Bayliss, D., Brahm, R., et al. 2015, AJ, 149, 166

Hartman, J. D., Bakos, G. Á, Béky, B., et al. 2012, AJ, 144, 139

Hellier, C., Anderson, D. R., Collier Cameron, A., et al. 2012, MNRAS, 426,739 
Hippler, S., Bergfors, C., Wolfgang, B., et al. 2009, Msngr, 137, 14 Howard, A. W., Marcy, G. W., Bryson, S. T., et al. 2012, ApJS, 201, 15 Janson, M., Durkan, S., Hippler, S., et al. 2017, A\&A, 599, A70 Jin, S., \& Mordasini, C. 2018, ApJ, 853, 163

Jordán, A., Brahm, R., Bakos, G. Á, et al. 2014, AJ, 148, 29

Kaufer, A., \& Pasquini, L. 1998, Proc. SPIE, 3355, 844

Knutson, H. A., Fulton, B. J., Montet, B. T., et al. 2014, ApJ, 785, 126

Kovács, G., Bakos, G., \& Noyes, R. W. 2005, MNRAS, 356, 557

Kovács, G., Zucker, S., \& Mazeh, T. 2002, A\&A, 391, 369

Kozai, Y. 1962, AJ, 67, 591

Li, G., Naoz, S., Kocsis, B., \& Loeb, A. 2014, ApJ, 785, 116

Lidov, M. L. 1962, P\&SS, 9, 719

Lin, D. N. C., Bodenheimer, P., \& Richardson, D. C. 1996, Natur, 380, 606 Mancini, L., Lillo-Box, J., Southworth, J., et al. 2016, A\&A, 590, A112 Mandel, K., \& Agol, E. 2002, ApJL, 580, L171

Mohler-Fischer, M., Mancini, L., Hartman, J. D., et al. 2013, A\&A, 558, A55

Mollière, P., \& Mordasini, C. 2012, A\&A, 547, A105

Mordasini, C., van Boekel, R., Mollière, P., Henning, T., \& Benneke, B. 2016, ApJ, 832, 41

Nagasawa, M., Ida, S., \& Bessho, T. 2008, ApJ, 678, 498

Neveu-VanMalle, M., Queloz, D., Anderson, D. R., et al. 2016, A\&A, 586, A93

Ngo, H., Knutson, H. A., Hinkley, S., et al. 2015, ApJ, 800, 138

Pál, A., Bakos, G. Á, Torres, G., et al. 2008, ApJ, 680, 1450
Penev, K., Bakos, G. Á, Bayliss, D., et al. 2013, AJ, 145, 5

Pepe, F., Ehrenreich, D., \& Meyer, M. R. 2014, Natur, 513, 358 Petrovich, C. 2015, ApJ, 805, 75

Queloz, D., Eggenberger, A., Mayor, M., et al. 2000, A\&A, 359, L13

Queloz, D., Mayor, M., Udry, S., et al. 2001, Msngr, 105, 1

Rabus, M., Alonso, R., Belmonte, J. A., et al. 2009, A\&A, 494, 391

Schlaufman, K. C. 2018, ApJ, 853, 37

Shporer, A., Zhou, G., Fulton, B. J., et al. 2017, AJ, 154, 188

Sozzetti, A., Torres, G., Charbonneau, D., et al. 2007, ApJ, 664, 1190

Telting, J. H., Avila, G., Buchhave, L., et al. 2014, AN, 335, 41

ter Braak, C. J. F. 2006, Statistics and Computing, 16, 239

Thorngren, D. P., Fortney, J. J., Murray-Clay, R. A., \& Lopez, E. D. 2016, ApJ, 831, 64

Triaud, A. H. M. J., Neveu-VanMalle, M., Lendl, M., et al. 2017, MNRAS, 467,1714

Vogt, S. S., Allen, S. L., Bigelow, B. C., et al. 1994, Proc. SPIE, 2198, 362

Wang, S., Addison, B., Fischer, D. A., et al. 2018, AJ, 155, 70

Weinberg, M. D., Yoon, I., \& Katz, N. 2013, arXiv:1301.3156

Winn, J. N., \& Fabrycky, D. C. 2015, ARA\&A, 53, 409

Yi, S., Demarque, P., Kim, Y.-C., et al. 2001, ApJS, 136, 417

Zhou, G., Bayliss, D., Hartman, J. D., et al. 2014a, MNRAS, 437, 2831

Zhou, G., Bayliss, D., Hartman, J. D., et al. 2015, ApJL, 814, L16

Zhou, G., Bayliss, D., Penev, K., et al. 2014b, AJ, 147, 144 